\title{
PONTOS CRÍTICOS DO PROCESSO DE PRODUÇÃO DE PISOS MACIÇOS DE MADEIRA
}

\author{
CRITICAL POINTS OF THE PRODUCTION PROCESS OF SOLID WOOD FLOORING
}

\author{
Philipe Ricardo Casemiro Soares ${ }^{1}$ Marcos Milan $^{2}$ Ivaldo Pontes Jankowsky ${ }^{3}$ \\ Antonio Jose da Silva Maciel ${ }^{4}$ Maria Raquel Kanieski ${ }^{5}$
}

\begin{abstract}
RESUMO
Os conceitos da qualidade representam um dos fatores importantes para o êxito das organizações, e dentre esses conceitos a estabilização dos processos de produção contribui para a melhoria, redução de desperdícios e aumento da competitividade. Com isso, o objetivo desse trabalho foi avaliar o processo de produção de pisos maciços de madeira de espécies comerciais quanto à sua previsibilidade e capacidade, com base em seus pontos críticos. Para tanto, a pesquisa foi dividida em três etapas. A primeira foi o mapeamento do processo da empresa e a elaboração de fluxogramas para as atividades. A segunda foi a identificação e a avaliação dos pontos críticos por meio da metodologia FMEA (Failure Mode and Effect Analysis) adaptada. A terceira foi a avaliação dos pontos críticos empregando o controle estatístico e a determinação da capacidade do processo pelo índice $\mathrm{C}_{\mathrm{pk}}$. Os resultados mostraram a existência de seis processos, sendo dois identificados como críticos. Nesses dois, quinze pontos foram considerados críticos e dois deles, relacionados com as dimensões das peças e defeitos provocados pela lixa, foram selecionados para avaliação. O processo produtivo da empresa é instável e não é capaz de produzir os pisos dentro das especificações e, portanto, essas especificações devem ser reavaliadas.
\end{abstract}

Palavras-chave: gestão da qualidade; capacidade de processo; pisos de madeira; FMEA.

\section{ABSTRACT}

The quality concepts represent one of the important factors for the success of organizations and among these concepts the stabilization of the production process contributes to the improvement, waste reduction and increased competitiveness. Thus, this study aimed to evaluate the production process of solid wood flooring on its predictability and capacity, based on its critical points. Therefore, the research was divided into three stages. The first one was the process mapping of the company and the elaboration of flowcharts for the activities. The second one was the identification and the evaluation of the critical points using FMEA (Failure Mode and Effect Analysis) adapted methodology. The third one was the evaluation of the critical points applying the statistical process control and the determination of the process capability for the $\mathrm{C}_{\mathrm{pk}}$ index. The results showed the existence of six processes, two of them are critical. In those two ones, fifteen points were considered critical and two of them, related with the dimension of the pieces and defects caused by sandpaper, were selected for evaluation. The productive process of the company is unstable and not capable to produce wood flooring according to the specifications and, therefore these specifications should be reevaluated.

Keywords: quality management; process capability; wood flooring; FMEA.

1. Engenheiro Florestal, MSc., Doutorando do Programa de Pós-graduação em Engenharia Florestal, Universidade Federal do Paraná, Av. Pref. Lothário Meissner, 900, CEP 80210-170, Curitiba (PR). prcsoares@gmail

2. Engenheiro Agrônomo, Dr., Professor Associado do Departamento de Biossistemas, Escola Superior de Agricultura "Luiz de Queiroz", Universidade de São Paulo, Caixa Postal 09, CEP 13418-900, Piracicaba (SP). macmilan@usp.br

3. Engenheiro Florestal, Dr., Professor Associado do Departamento de Ciências Florestais, Escola Superior de Agricultura "Luiz de Queiroz", Universidade de São Paulo, Caixa Postal 09, CEP 13418-900, Piracicaba (SP). jankowsky@usp.br

4. Engenheiro Agrícola, Dr., Professor Assistente da Faculdade de Engenharia Agrícola, Universidade Estadual de Campinas, Av. Cândido Rondon, 501, CEP 13083-875, Campinas (SP). amaciel@feagri.unicamp.br

5. Engenheira Florestal, Msc., Doutoranda do Programa de Pós-graduação em Engenharia Florestal, Universidade Federal do Paraná, Av. Pref. Lothário Meissner, 900, CEP 80210-170, Curitiba (PR). raquelkanieski@gmail.com Recebido para publicação em 18/10/2010 e aceito em 10/05/2011 


\section{INTRODUÇÃO}

O conceito da qualidade se tornou um fator importante na administração das organizações para atender às exigências dos mercados. A implantação de programas de qualidade tem como benefícios a estabilização dos processos de produção reduzindo as não conformidades e, consequentemente, os custos, resultando em um aumento na competitividade. Em uma pesquisa realizada com empresas de grande porte do Brasil, Pinto et al. (2006) concluíram que os investimentos realizados na implantação e manutenção desses programas são diretamente proporcionais à satisfação dos clientes $\mathrm{e}$ aumentam o retorno financeiro. Entretanto, para que os esforços de implantação sejam bem sucedidos, a adoção deve ser realizada de maneira sistêmica e com o envolvimento das pessoas em todos os níveis organizacionais (TRINDADE et al., 2000).

Um dos princípios dos programas é o de que a organização pode ser dividida, hierarquicamente, em três grandes áreas: estratégica, tática e operacional (rotina). A área estratégica é a responsável pelos rumos e/ou estratégias, a tática pelo desenvolvimento das estratégias e a rotina pela definição dos meios e recursos para a execução das tarefas. A estabilização da rotina é um dos principais fatores que permite que uma organização atinja e mantenha um padrão desejado dos seus produtos. A ausência desse padrão dificulta e pode impedir a implantação de melhorias no processo, afetando a competitividade.

Para a economia brasileira o setor florestal brasileiro é de grande importância. No ano de 2007 ele movimentou um montante de U\$ 44,6 bilhões, representando $3,5 \%$ do PIB do país. Dentro desse setor, o segmento de pisos de madeira no Brasil vem apresentando um crescimento constante de produção e consumo atingindo entre os anos de 1999 e $2008140 \%$ e $187 \%$ de aumento, respectivamente (ABIMCI - ASSOCIAÇÃO BRASILEIRA DA INDÚSTRIA DE MADEIRA PROCESSADA MECANICAMENTE, 2009). Em 2008, segundo a (ANPM) Associação Nacional dos Produtores de Pisos de Madeira (2009, não publicado) a produção de pisos atingiu 28,2 milhões de $\mathrm{m}^{2}$, movimentando US\$ 919,8 milhões. Apesar da importância econômica e a necessidade de manter a competitividade em longo prazo, um grande número de empresas, especialmente as de médio e pequeno porte, ainda não se atentaram para a importância dos conceitos contidos nos programas de qualidade.
As que já identificaram essas oportunidades de melhorias utilizam os conceitos na indústria e/ou nas atividades silviculturais (JACOVINE et al., 2005).

Apesar da aplicação dos conceitos da qualidade no setor florestal ainda ser incipiente, a preocupação com esses conceitos é antiga, sendo que o primeiro sistema de vistoria da qualidade foi implementado no Estado de São Paulo, na década de 1980 (REZENDE etal., 2000). Destacam-se também os trabalhos realizados por Freitas et al. (1980), com a aplicação do controle de qualidade nas diferentes atividades silviculturais em uma floresta de Eucalyptus: implantação, manutenção, exploração e desbrota; Rezende et al. (2000) e Jacovine et al. (2005), avaliando a colheita florestal; Jacovine et al. (1999) e Leite et al. (2005), analisando os custos da qualidade em diferentes atividades; Milan et al. (2003) que aplicaram o método QFD (Quality Function Deployment) para o planejamento da qualidade, estabelecendo as prioridades no preparo de solo a partir da exigência das mudas de eucalipto (Eucalyptus spp.). Nas indústrias de processamento da madeira, Matos e Milan (2009) desenvolveram indicadores de desempenho para empresa de pequeno porte do setor de portas e esquadrias, utilizando a metodologia FMEA (Failure Mode and Effect Analysis), e Bonduelle (2000) aplicou a técnica de planejamento de experimentos para melhoria do produto de uma empresa de chapas de fibras de madeira.

Destacando-se a importância dos programas de qualidade para o êxito das organizações e considerando-se a necessidade de estabilizar o processo de produção para a melhoria, redução de desperdícios e aumento da competitividade, o objetivo desse trabalho foi avaliar o processo de produção de pisos maciços de madeira de espécies comerciais quanto à sua previsibilidade $\mathrm{e}$ capacidade, com base em seus pontos críticos.

\section{MATERIAL E MÉTODOS}

O trabalho foi desenvolvido em uma empresa do setor de pisos maciços de madeira, localizada no município de Tietê, no estado de São Paulo. A empresa é atualmente a maior do setor em toda a América Latina, contando com cerca de 500 funcionários diretos, distribuídos em duas plantas, com capacidade produtiva mensal de $130.000 \mathrm{~m}^{2}$ de pisos, sendo, aproximadamente, $80 \%$ desse total destinado à exportação, principalmente para Itália 
e Estados Unidos. A pesquisa foi realizada em três etapas sequenciais: mapeamento e elaboração do fluxograma do processo; identificação e avaliação dos setores e pontos críticos; avaliação do processo. A escolha das espécies comerciais utilizadas no trabalho foi realizada em acordo com a empresa, que considera as necessidades de produção e a incidência de defeitos.

\section{Mapeamento do processo}

O mapeamento do processo de produção foi realizado por meio da divisão da empresa em setores principais, de acordo com o fluxo de material e observando-se a relação cliente/fornecedor. Após a divisão, os setores foram analisados e as atividades por eles desenvolvidas foram registradas. Os fluxogramas do mapeamento foram elaborados com base nos símbolos apresentados por Trindade et al. (2000).

\section{Identificação dos pontos críticos dos processos}

$\mathrm{Na}$ segunda etapa do trabalho realizou-se a identificação e avaliação dos pontos críticos do processo em quatro subetapas: determinação dos setores críticos; identificação dos pontos críticos; determinação dos potenciais de falha; avaliação dos pontos críticos.

Para a determinação dos setores responsáveis pelos maiores riscos durante o processamento da madeira, utilizou-se a metodologia (FMEA) - Failure Mode and Effect Analysis, adaptada de Stamatis (1995). Para isso, cada setor foi classificado quanto à ocorrência $(\mathrm{Oc})$, severidade $(\mathrm{Sv})$ e detecção $(\mathrm{Dt})$ de falhas em escala de 1 a 5 . Os índices utilizados e os critérios de classificação foram empregados de acordo com a proposta de Matos e Milan (2009). A partir dos valores atribuídos a cada critério, calculou-se o índice de risco (IR) de cada setor pela equação:

$$
I R=O c \times S v \times D t
$$

Em que: $\mathrm{Oc}=$ índice de ocorrência; $\mathrm{Sv}=$ índice de severidade; $\mathrm{Dt}=$ índice de detecção

Com isso, os setores foram classificados quanto ao seu potencial de risco, com base no maior índice (IR). A identificação dos pontos críticos e a avaliação dos potenciais de falhas nos setores selecionados foram realizadas por meio de entrevistas individuais com funcionários. Além disso, pessoas externas aos setores, com visão geral do processo, também foram consultadas sobre as possíveis falhas. Na avaliação dos pontos críticos, os entrevistados deram notas aos critérios ocorrência, severidade e detecção dos itens, em escala de 1 a 5 , com base na classificação apresentada por Matos e Milan (2009). Com os resultados, calculou-se o índice de risco, da mesma forma como foi feito para os setores. Considerou-se também, para a seleção dos pontos, a disponibilidade de recursos na empresa e a dificuldade para obtenção de dados. A decisão foi tomada em comum acordo com a empresa.

\section{Avaliação do processo}

Cada setor e ponto críticos selecionados foram avaliados por meio das técnicas do controle estatístico do processo. O controle por variáveis foi utilizado quando o ponto crítico era caracterizado como uma variável contínua. A metodologia teve início com a mensuração do indicador referente ao ponto crítico, com o tamanho das amostras (n) e a frequência de amostragem, determinados em função da produtividade do setor e recomendações da bibliografia específica (OAKLAND, 2003; MONTGOMERY, 2004). Com base nos dados obtidos para cada ponto crítico, a dispersão foi avaliada por meio do histograma. A estabilidade do processo foi analisada por meio da carta de controle (média e dispersão), de acordo com as metodologias apresentadas por Oakland (2003) e Montgomery (2004).

Para o gráfico por média e dispersão, calculou-se a linha média (LM), o limite superior de controle (LSC) e o limite inferior de controle (LIC), segundo equações propostas por Oakland (2003). Às cartas de controle por média foram acrescentados os limites de especificação superior (LSE) e inferior (LIE), estabelecidos pela empresa. Nos gráficos foram inseridos os valores médios e o desvio padrão de cada amostra, respectivamente, para os gráficos de média e de dispersão, com o objetivo de verificar a variabilidade das variáveis estudadas e, consequentemente, a estabilidade do processo. Para isso observou-se a existência de pontos fora dos limites de atenção, além de tendências na distribuição dos dados.

O controle por atributos foi utilizado quando o ponto crítico foi caracterizado como uma variável discreta, observando-se o número de não conformidades. O tamanho das amostras (n) e a frequência de amostragem foram determinados em função da produtividade do setor. Para a avaliação dos atributos, o tipo de carta de controle escolhido 
foi o gráfico- $n p$ para número de unidades defeituosas ou não conformes. De acordo com Oakland (2003), o gráfico- $n p$ é elaborado de maneira mais simples e conveniente, por utilizar os dados brutos, sem a necessidade do cálculo da probabilidade de ocorrência de defeitos. Na elaboração desses gráficos foram calculados apenas a linha média (LM) e o limite superior de controle (LSC), a partir das equações apresentadas por Oakland (2003). A quantidade de defeitos de cada amostra foi inserida nos gráficos para a avaliação da estabilidade do processo, verificando-se tendências e pontos fora dos limites.

Para a confecção das cartas de controle é necessário que o processo seja considerado estável. Se essa condição não fosse obtida, ajustavam-se os dados originais, eliminando-se os pontos com fontes especiais de variação e recalculando-se todas as linhas de controle, conforme a metodologia descrita por Oakland (2003) e Montgomery (2004).

\section{Capacidade do Processo}

Para as variáveis contínuas determinou-se a capacidade do processo, a partir do índice $\mathrm{C}_{\mathrm{pk}}$, sendo considerado o menor valor obtido entre $\mathrm{C}_{\mathrm{pk}}$ superior e inferior, calculados pelas equações apresentadas por Montgomery (2004). Um valor de $\mathrm{C}_{\mathrm{pk}}$ menor ou igual a um (1) indica que o processo é incapaz de produzir dentro das especificações da empresa, ou seja, o desvio padrão da variável é maior, ou não está centralizado no intervalo especificado. Valores entre um (1) e dois (2) indicam que o processo é capaz de produzir dentro das especificações, no entanto, ações deverão ser tomadas para a detecção das não conformidades, e valores de $\mathrm{C}_{\mathrm{pk}}$ acima de dois (2) indicam um alto nível de confiança no processo (OAKLAND, 2003).

\section{RESULTADOS E DISCUSSÃO}

Por meio do mapeamento identificaramse seis processos na produção dos pisos de madeira: recebimento, secagem, processamento primário, processamento secundário, acabamento e embalagem. Na Figura 1 é possível observar toda a sequência desde a chegada da matériaprima, madeira, à empresa (recebimento) até a embalagem do produto final. A matéria-prima não segue um fluxo contínuo, sendo estocada após beneficiamento, junto ao próprio equipamento ou em galpões.

No processo recebimento, que ocorre nos pátios, são realizados a recepção da matériaprima, verificação e preparo para a pré-secagem. O planejamento da produção se inicia no processo secagem e tem como função disponibilizar aos demais setores a madeira seca de acordo com as especificações dos clientes.

$\mathrm{O}$ setor processamento primário é responsável pelo primeiro beneficiamento. A madeira é serrada, aplainada, tem os seus defeitos removidos e encaminhada para o segundo beneficiamento (processamento secundário), que dá forma ao piso, com encaixes confeccionados e dimensões próximas às especificadas.

No processo acabamento, o piso pode seguir diferentes caminhos, de acordo com o acabamento. Quando comercializado na forma natural, as peças são somente lixadas. No caso de pisos envernizados, as peças passam por sequências de lixas, aplicação de seladora ou verniz e secagem. A sequência de produção dos pisos tingidos difere dos envernizados pela aplicação de tinta e isolante, intercalada por escovação e seguida de secagem. O último processo (embalagem) é responsável pela colocação dos pisos nas caixas e a expedição do produto sendo realizado manualmente em quase sua totalidade.

\section{Identificação dos pontos críticos dos processos}

Para a identificação dos pontos críticos, inicialmente selecionou-se os processos críticos por meio de entrevistas realizadas com funcionários da empresa. Os resultados são apresentados na Tabela 1.

Na Tabela 1 é possível identificar que o processo de acabamento possui o maior índice médio de ocorrência de defeitos $(3,8)$, apresentando também um alto índice de severidade $(3,8)$. A severidade foi maior para o setor processamento secundário $(4,8)$, indicandoque as falhas que ocorrem nessa etapa afetam negativamente, em muito, a relação com o cliente. Além disso, o acabamento e o processamento secundário apresentaram maiores índices de risco, respectivamente 40,4 e 33,6, justificando a escolha dos mesmos para a sequência do estudo. Com relação à detecção das falhas, o processo processamento primário é o que apresenta o maior índice $(3,3)$, por possuir um controle menos rigoroso que os demais processos da empresa. $\mathrm{O}$ processo de secagem da madeira foi o que apresentou menor índice de risco médio $(13,0)$, resultante da baixa ocorrência e da melhor detecção das falhas por apresentar um monitoramento constante. Entretanto, o índice de severidade é 


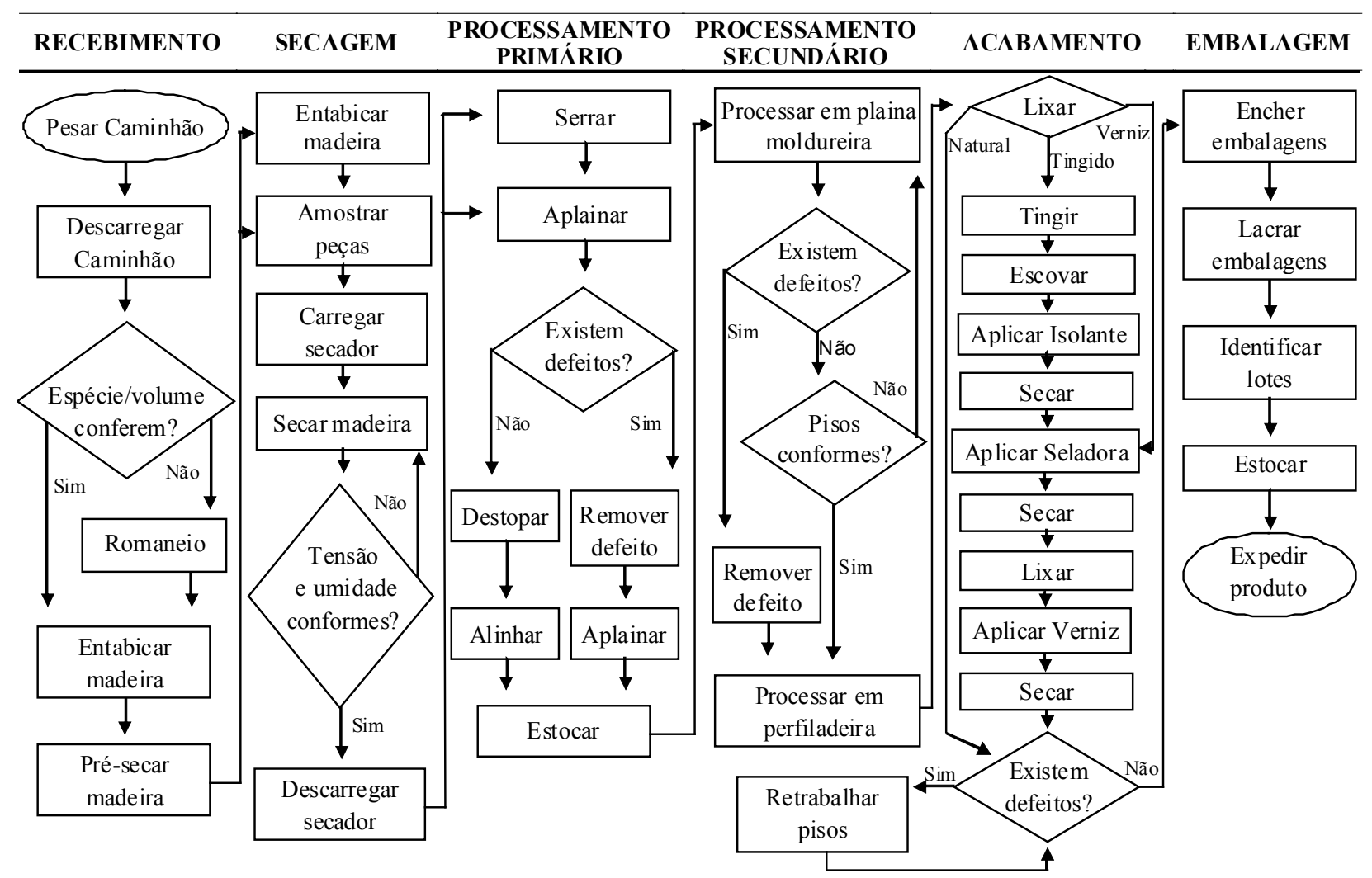

FIGURA 1: Mapeamento do processo e fluxograma de atividades na produção de pisos maciços de madeira. FIGURE 1: Process mapping and activities flowcharts in the manufacture of solid wood flooring.

TABELA 1: Classificação dos processos pelo índice de risco com base na metodologia FMEA TABLE 1: Classification of processes by risk priority number, based on FMEA adapted methodology.

\begin{tabular}{lcccc}
\hline \multirow{2}{*}{\multicolumn{1}{c}{ Processo }} & \multicolumn{3}{c}{ Critério } & \multirow{2}{*}{ IR } \\
\cline { 2 - 4 } & Oc & Sv & Dt & \\
\hline Recebimento & 2,8 & 3,3 & 2,5 & 23,1 \\
Secagem & 1,8 & 4,0 & 1,8 & 13,0 \\
Processamento Primário & 2,0 & 4,0 & 3,3 & 26,4 \\
Processamento Secundário & 2,8 & 4,8 & 2,5 & 33,6 \\
Acabamento & 3,8 & 3,8 & 2,8 & 40,4 \\
Embalagem & 2,5 & 3,5 & 3,0 & 26,3 \\
\hline
\end{tabular}

Em que: $\mathrm{Oc}=$ ocorrência; $\mathrm{Sv}=$ severidade; $\mathrm{Dt}=$ Detecção; $\mathrm{IR}=$ Índice de risco.

considerado alto, significando que, se alguma falha não for detectada, o cliente certamente perceberá.

Com a seleção dos processos críticos a próxima etapa foi identificar e avaliar os pontos críticos de cada um. A identificação e a avaliação dos pontos críticos ocorreram por meio de entrevistas com funcionários de cada setor, além de pessoas externas com visão geral do processo. Realizaramse dezoito entrevistas no setor processamento secundário e dez no de acabamento. Os resultados são apresentados na Tabela 2.

De acordo com a Tabela 2, o principal potencial de falha no processamento secundário é a mistura de madeiras nos lotes ( $I R=18,2)$. No entanto esse problema não é gerado diretamente, tendo a origem nos processos anteriores, especialmente no recebimento. O mesmo acontece com relação ao ponto crítico "peças com defeitos naturais da 
TABELA 2: Identificação dos pontos críticos dos processos pelo índice de risco, com base na metodologia FMEA adaptada.

TABLE 2: Identification of the process critical points by risk priority number, based on FMEA adapted methodology.

\begin{tabular}{clrrrr}
\hline \multirow{2}{*}{ Processo } & \multicolumn{1}{c}{ Ponto Crítico } & \multicolumn{3}{c}{ Critério } & \multirow{2}{*}{ IR } \\
\cline { 3 - 5 } & & Oc & Sv & Dt & \\
\hline \multirow{3}{*}{$\begin{array}{c}\text { Processamento } \\
\text { Secundário }\end{array}$} & Peças com defeitos naturais da madeira & 3,1 & 2,4 & 2,1 & 15,6 \\
& Peças fora das dimensões especificadas & 2,4 & 2,6 & 2,4 & 15,0 \\
& Peças fora de esquadro & 2,2 & 2,7 & 2,6 & 15,4 \\
& Peças marcadas pelas facas de corte & 2,7 & 1,6 & 2,2 & 9,5 \\
& Lotes com mistura de madeiras & 3,6 & 2,3 & 2,2 & 18,2 \\
\hline \multirow{5}{*}{ Acabamento } & Peças com defeitos naturais da madeira & 2,3 & 2,1 & 1,7 & 8,2 \\
& Peças queimadas pela secagem ultravioleta & 1,7 & 2,9 & 2,3 & 11,3 \\
& Verniz não adere corretamente & 2,1 & 2,1 & 2,4 & 10,6 \\
& Peças marcadas pela lixa & 2,2 & 2,9 & 2,3 & 14,7 \\
& Peças fora das dimensões especificadas & 2,0 & 2,7 & 2,4 & 13,0 \\
& Peças com falha no verniz & 2,8 & 2,6 & 1,9 & 13,8 \\
& Peças com brilho fora do padrão & 1,8 & 2,3 & 1,8 & 7,5 \\
& Peças com manchas de extrativos da madeira & 3,5 & 2,9 & 2,4 & 24,4 \\
& Peças com coloração incorreta & 2,0 & 3,1 & 2,0 & 12,4 \\
& Peças que param a linha de acabamento & 2,1 & 2,6 & 1,7 & 9,3 \\
\hline
\end{tabular}

Em que: $\mathrm{Oc}=$ ocorrência; $\mathrm{Sv}=$ severidade; $\mathrm{Dt}=$ Detecção; $\mathrm{IR}=$ Índice de risco.

madeira". Os principais problemas resultantes são as peças fora das dimensões especificadas e de esquadro com IR de 15,0 e 15,4 , respectivamente. Apesar dos menores valores de ocorrência, eles possuem maiores índices de severidade e a possibilidade de detecção não é alta, o que significa que a falha é relativamente severa e pode não ser detectada chegando aos clientes, afetando o relacionamento com a empresa.

No processo acabamento, o maior índice de risco observado foi para o ponto crítico "peças com manchas do extrativo da madeira" $(24,4)$. Esse ponto não foi selecionado para a avaliação por ser um defeito natural da espécie processada. Com relação às falhas geradas pelo processo, destacamse as peças marcadas pela lixa, com falha no verniz e fora das dimensões especificadas, com índice de risco (IR) de 14,7, 13,8 e 13,0, respectivamente, e altos valores para a severidade. Isso significa que se a falha ocorrer, ela será de natureza severa e se não for detectada durante o processo, o cliente perceberá.

Com base nesses resultados e em comum acordo com a empresa, os pontos críticos selecionados para a próxima etapa da pesquisa foram as peças fora de esquadro (IR 15,4) para o setor de processamento secundário e as peças marcadas pela lixa $(14,7)$ para o acabamento.

\section{Avaliação do processo}

Nessa etapa, analisou-se o trabalho de uma plaina moldureira e uma das linhas de acabamento da empresa, referentes aos pontos críticos peças fora de esquadro e peças marcadas pela lixa, respectivamente. No setor processamento secundário, o trabalho da plaina foi avaliada por meio do controle estatístico do processo por variáveis, durante o processamento da espécie cumaru e a variável observada corresponde à largura dos pisos. No processo acabamento, empregou-se o controle estatístico por atributos, para a análise de peças marcadas pela lixa, durante o processamento de pisos da espécie amendoim.

\section{Ponto crítico: peças fora de esquadro}

Realizou-se a amostragem de sete pisos de cumaru a cada intervalo de 30 minutos, mensurandose a largura das peças em dois pontos, para obtenção de um valor médio, totalizando 71 amostras (497 pisos). Os resultados são apresentados na Figura 2. Os limites especificados pela empresa, inferior e superior, correspondem a 94,90 e $95,19 \mathrm{~mm}$, respectivamente, e aproximadamente de $35 \%$ dos dados de largura, apenas, estão entre os limites 
desejados.

Os gráficos de controle por média (A) e dispersão (B) para a variável largura são apresentados na Figura 3. Na figura pode-se observar que o processo é instável por apresentar pontos que ultrapassam os limites de controle, superior e inferior.

O gráfico de controle por média (Figura 3A) mostra que no início a média estava acima da linha superior de controle. Um ajuste da máquina referente à largura das peças no valor de $-0,08 \mathrm{~mm}$, entre as amostras 3 e 4 , resultou no decréscimo da média, mantendo-se dentro dos limites até a amostra 9, onde o encaixe do tipo fêmea foi ajustado em $0,1 \mathrm{~mm}$. Esse fato pode ter gerado um novo decréscimo nos valores da média, atingindo pontos abaixo do limite inferior de controle. As fresas de corte foram substituídas, entre as amostras 17 e 18, ocasionando um acréscimo na média, que se manteve dentro dos limites até o ponto 27 , a partir do qual passou a ser processada madeira de cumaru com coloração mais avermelhada. Esse fato pode ter alterado a densidade da madeira, mesmo que não tenha havido mudança na espécie, o que alterou novamente a média das amostras. Durante todo o período de avaliação, a média esteve próxima ao limite inferior de especificação, na maioria dos casos abaixo dele, indicando uma provável falta de aferição do equipamento de medição utilizado pela empresa.
O gráfico de controle por dispersão mostra que a variação do desvio padrão das amostras foi pequena, com exceção de 2 pontos (5 e 10), que ficaram fora dos limites, podendo ser resultado dos ajustes realizados. A análise conjunta dos gráficos mostra um processo que sofre pouca variação, mas distante do valor alvo especificado pela empresa. Portanto, para esta situação, ajustar o processo para que ele permaneça dentro dos limites especificados é a principal ação a ser tomada.

Para obtenção das cartas de controle e cálculo da capacidade do processo, inicialmente eliminou-se 12 amostras que apresentaram causas especiais, de acordo com a proposta de Oakland (2003) e Montgomery (2004), recalculando-se os limites de controle. As cartas de controle por média (A) e dispersão (B) para a variável são apresentadas na Figura 4.

Com relação à capacidade do processo, os índices $\mathrm{C}_{\mathrm{pk}}$ calculados foram 5,1 e $-0,4$, respectivamente, para $\mathrm{C}_{\mathrm{pk}}$ superior e inferior. Sendo o menor valor obtido inferior a 1, é possível afirmar que a plaina avaliada, nas condições atuais, é incapaz de produzir pisos de cumaru, dentro das especificações, ou seja, a variabilidade do processo é maior que a variação especificada pela empresa. Nesse caso, a máquina deve sofrer ajustes para atender aos limites especificados pela empresa, ou esses limites devem ser reavaliados quanto a sua necessidade.

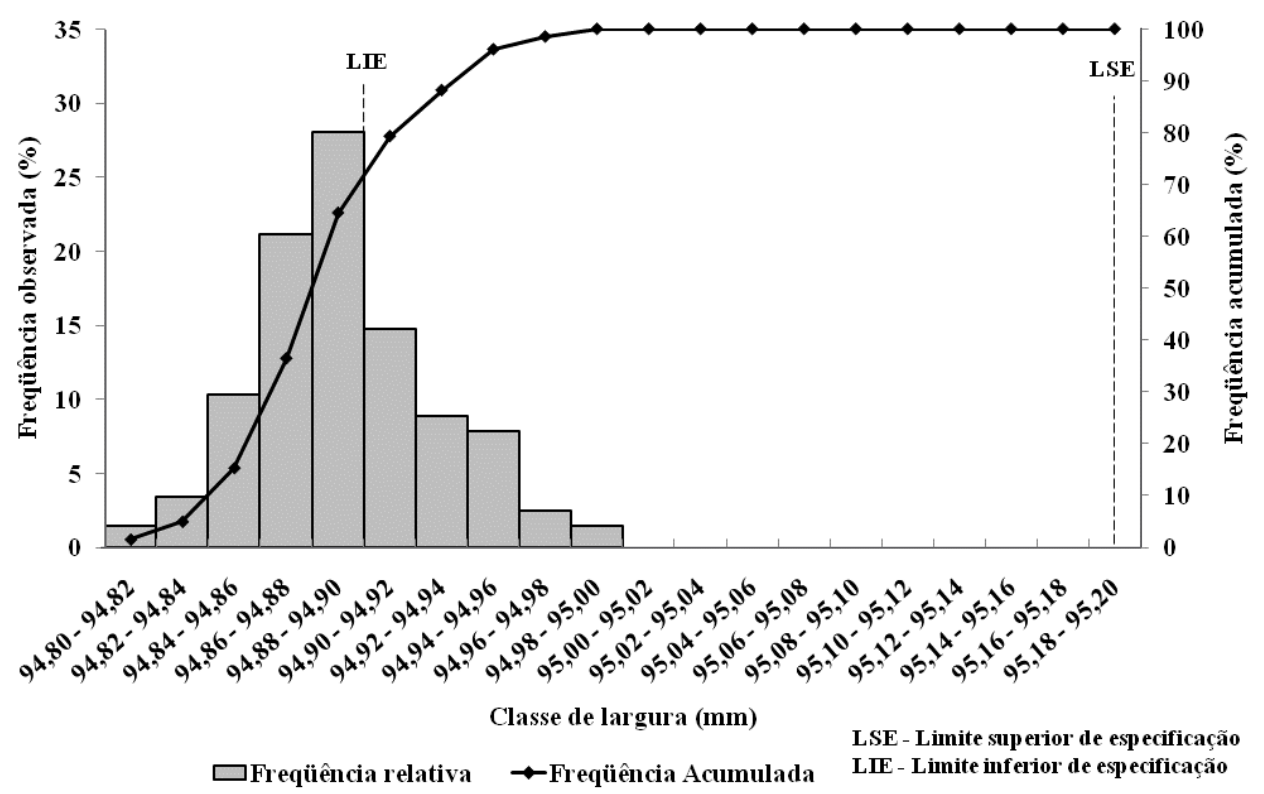

FIGURA 2: Distribuição dos dados referentes à largura do piso de cumaru.

FIGURE 2: Data distribution of the width of cumaru flooring. 

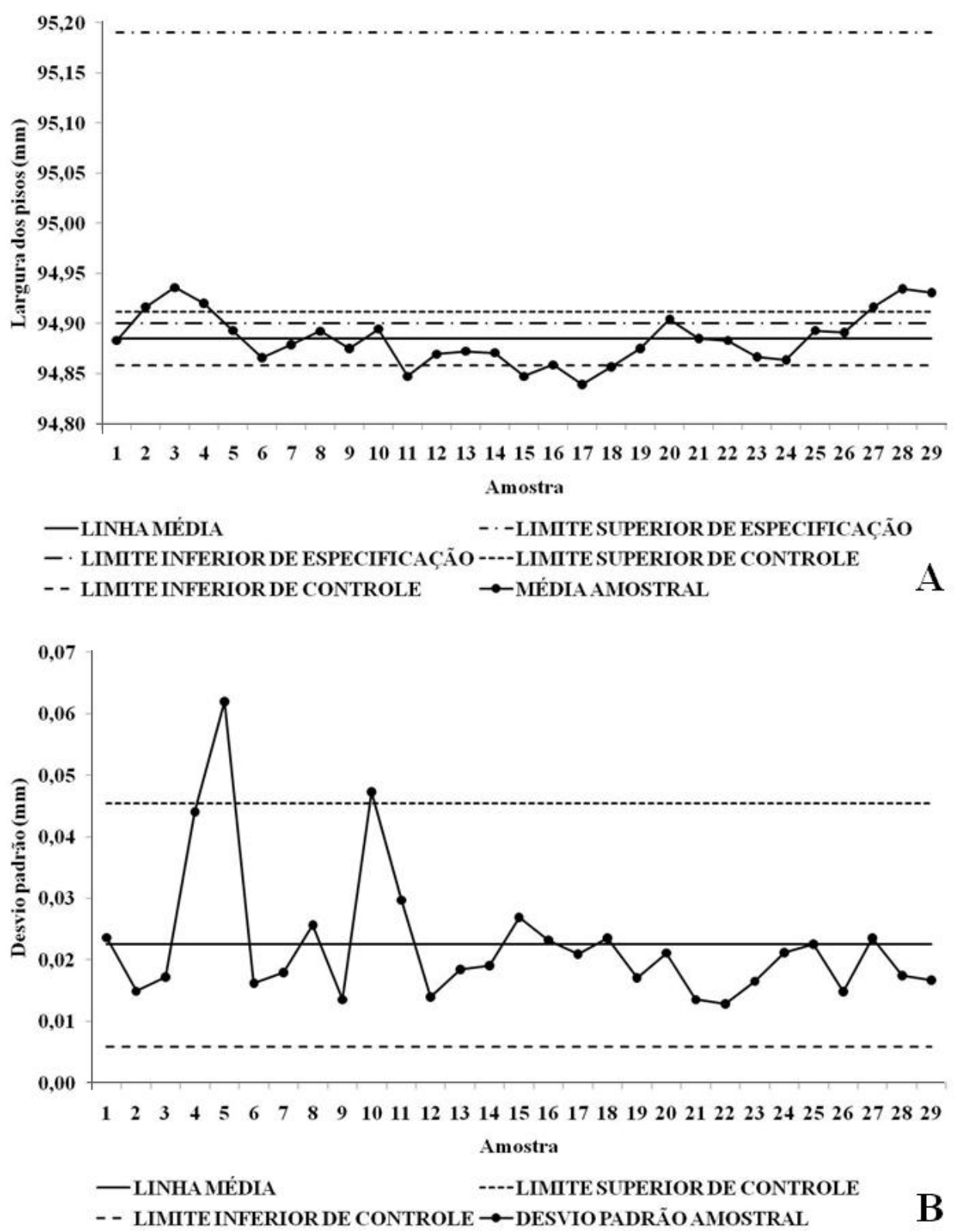

FIGURA 3: Variação da largura do piso de cumaru: gráficos de controle por média (A) e por dispersão (B). FIGURE 3: Variation in the width of cumaru flooring: control graphs for mean (A) and standard derivation (B).

\section{Ponto crítico: peças marcadas pela lixa}

Analisou-se uma das linhas durante processamento de pisos de amendoim. Cada amostra era composta por 100 peças, com frequência de amostragem de 10 minutos. Os resultados referentes ao atributo, peças marcadas no processo, são apresentados na Figura 5. Observa-se que o número de não conformidades para o atributo variou de 0 a 13 , e que $60 \%$ das amostras, aproximadamente, estão entre 0 e 1 defeitos.
O gráfico-np é apresentado na Figura 6. O processo é instável, expondo diversos pontos acima do limite de controle, situação esta causada pela parada na linha, o que expõe as peças a um maior tempo sob as lixas.

Eliminando-se 9amostras, que apresentaram causas especiais de variação (OAKLAND, 2003; MONTGOMERY, 2004) a média de defeitos reduziu para um número entre 1 e 2 , para cada 100 peças, enquanto o limite superior de controle, entre 4 e 5 unidades defeituosas (Figura 7). 


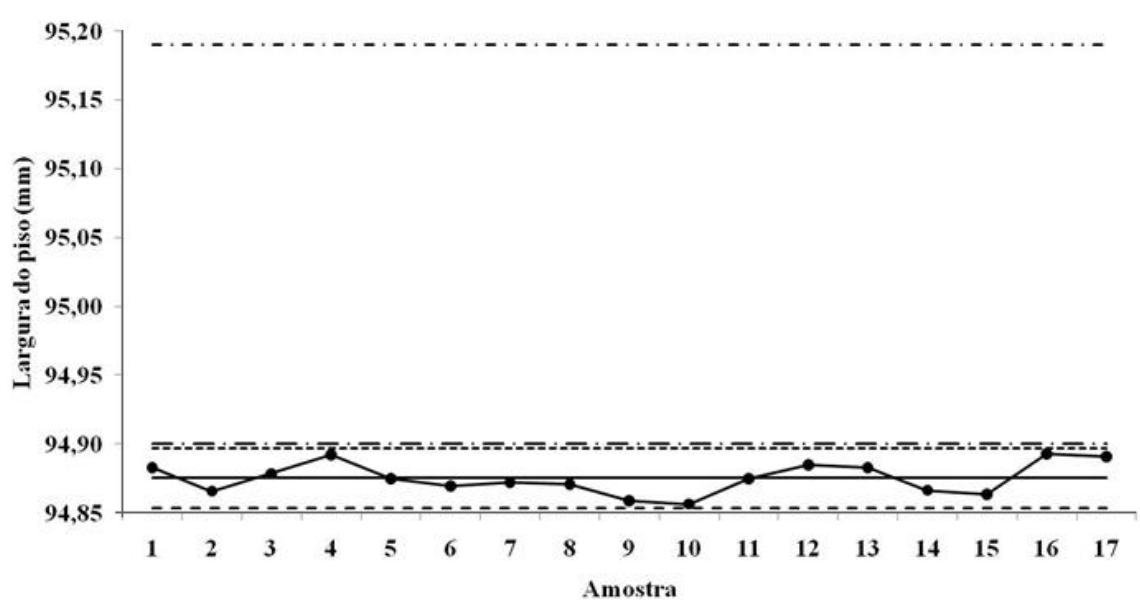

- LINHA MÉDIA - - - LIMITE SUPERIOR DE ESPECIFICACC̃̃O

- LIMITE INFERIOR DE ESPECIFICACÃO ----LIMITE SUPERIOR DE CONTROLE

- - LIMITE INFERIOR DE CONTROLE $\rightarrow$ MÉDIA AMOSTRAL

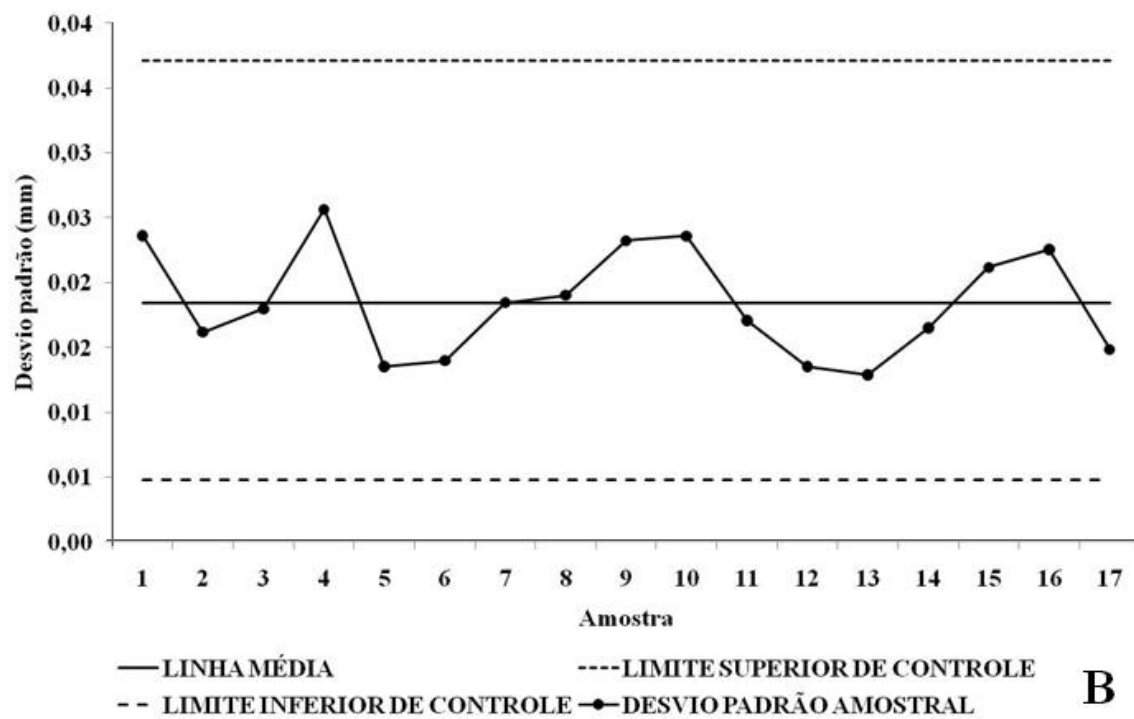

FIGURA 4: Cartas de controle por média (A) e dispersão (B) para a largura do piso de cumaru FIGURE 4: Control charts for mean (A) and standard derivation (B) for the width of cumaru flooring.

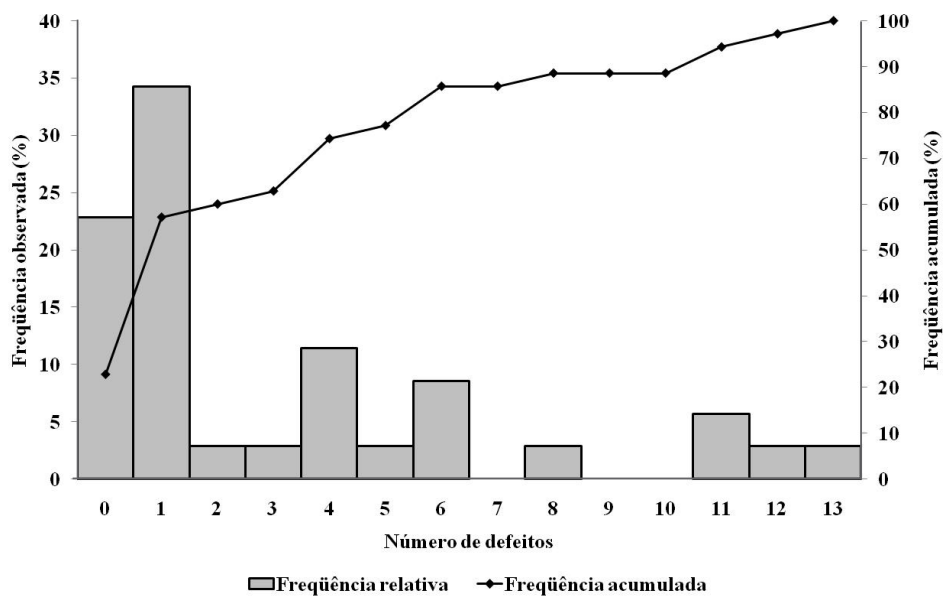

FIGURA 5: Pisos de madeira de amendoim marcados pela lixa em amostras de 100 peças.

FIGURE 5: Amendoim wood flooring marked by the sandpaper in samples of 100 pieces. 


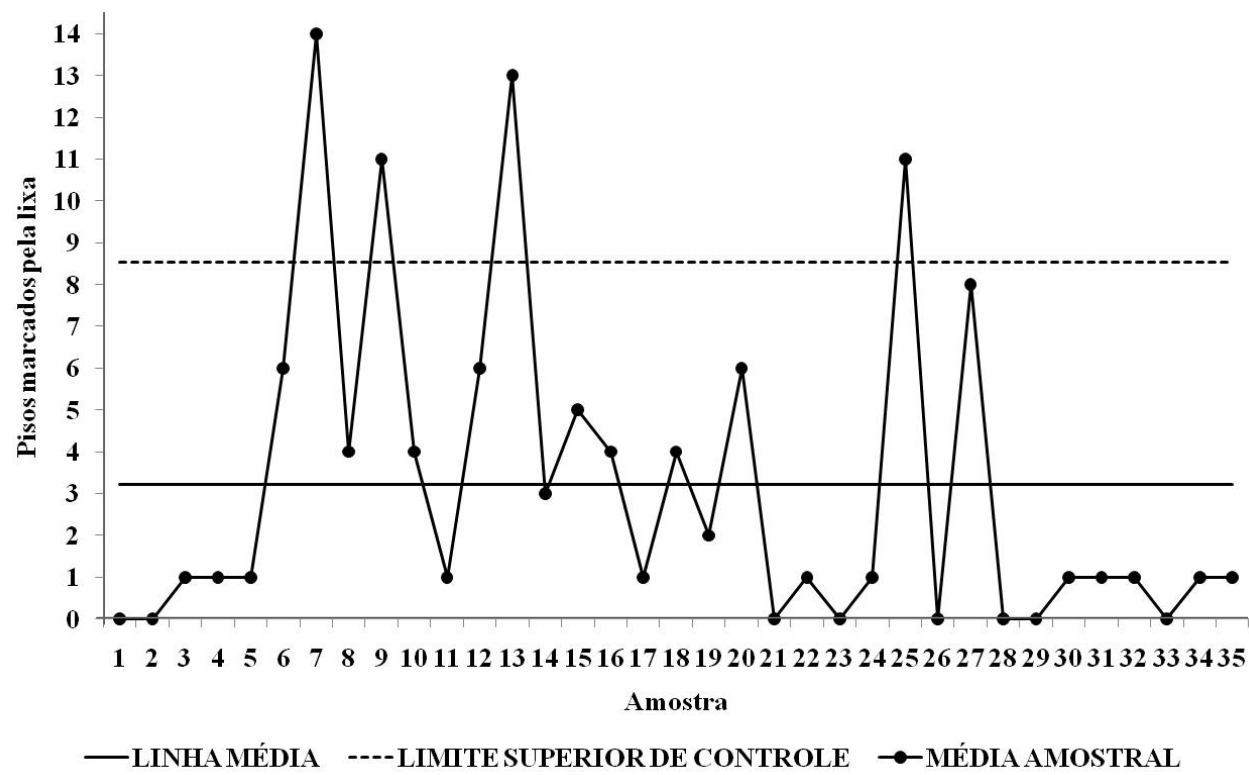

FIGURA 6: Gráfico-np referente às peças marcadas pela lixa (madeira:amendoim). FIGURE 6: Np-charts for amendoim flooring marked by the sandpaper.

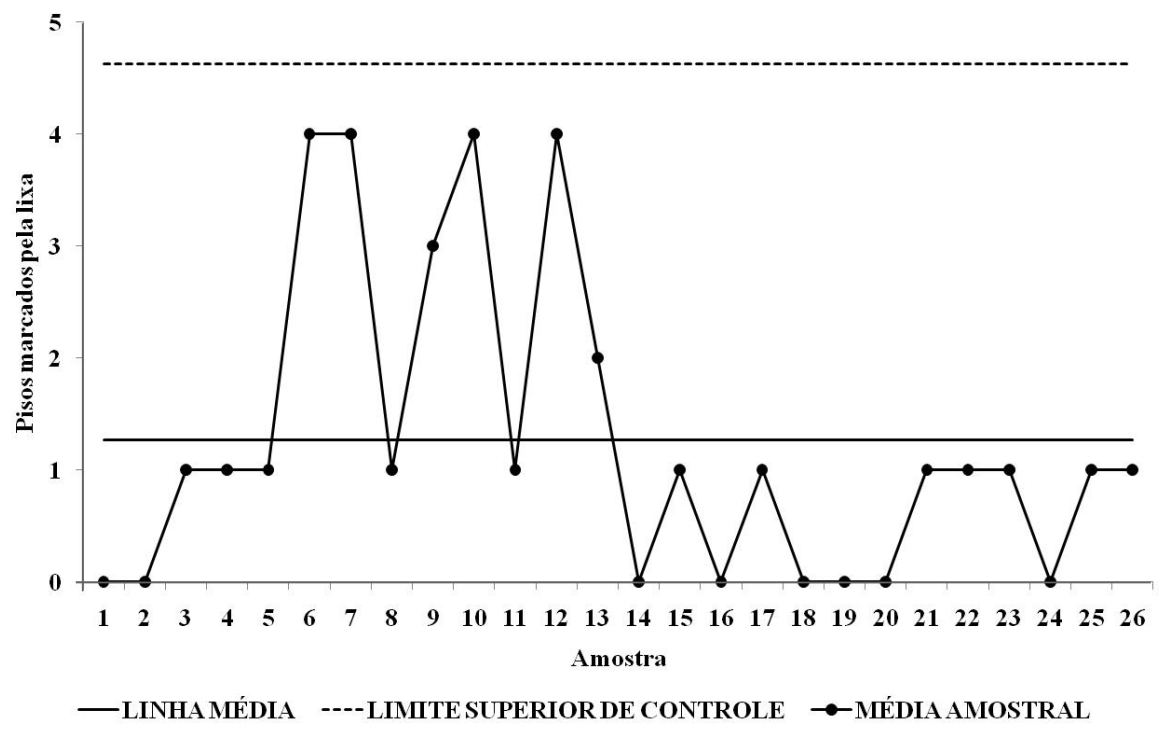

FIGURA 7: Gráfico-np de controle para pisos de amendoim marcados pela lixa.

FIGURE 7: Control np-charts for amendoim flooring marked by the sandpaper.

\section{CONCLUSÕES}

Dos seis processos, dois foram avaliados como os mais críticos (processamento secundário e acabamento) e esses apresentaram quinze pontos que, com os índices de riscos apresentados, podem afetar a escolha do produto pelo cliente, influenciando na competitividade.

Os esforços para a garantia da qualidade do produto que chegam ao cliente são concentrados na inspeção de unidades para a detecção de não conformidades, com pequeno número de ações tomadas para a prevenção da ocorrência de defeitos.

Os pontos críticos analisados (dimensão das peças e defeitos gerados pela lixa) foram considerados como instáveis e não previsíveis; é preciso rever os processos para estabilizar a rotina, um dos principais fatores para manter um padrão desejado dos produtos.

Para minimizar as ocorrências, a empresa deve realizar o estudo da causa raiz de cada situação indesejada e desenvolver meios para bloqueá-las. 
A adoção da gestão por processos, estabelecendo a relação cliente/fornecedor interno, também pode contribuir para a melhoria. A empresa deve alterar suas estratégias de garantia da qualidade, adotando a gestão por processos.

\section{REFERÊNCIAS BIBLIOGRÀFICAS}

ABIMCI - Associação Brasileira da Indústria de Madeira Processada Mecanicamente. Estudo setorial 2009 ano base 2008: Indústria de madeira processada mecanicamente. Curitiba, 2009. $43 \mathrm{p}$.

ANPM - Associação Nacional dos Produtores de Pisos de Madeira. Estatísticas referentes ao setor de pisos de madeira no Brasil. Piracicaba, 2009. Não publicado.

BONDUELLE, G. M. Aplicação do planejamento de experimentos no controle da fabricação de chapas de fibras de madeira. Cerne, Lavras, v. 6, n. 2, p. 1-10, 2000.

CAMPOS, V. F. Gerenciamento da rotina do trabalho do dia-a-dia. Nova Lima: INDG, 2004. $266 \mathrm{p}$.

FREITAS, M. et al. Avaliação e controle de qualidade em florestas de Eucalyptus. Circular Técnica IPEF, Piracicaba, n. 91, 8 p., fev. 1980. JACOVINE, L. A. G. et al. Avaliação da qualidade operacional em cinco subsistemas de colheita florestal. Revista Árvore, Viçosa, v. 29, n. 3, p. 391-400, maio/jun. 2005.

JACOVINE, L. A. G. et al. Descrição e uso de uma metodologia para avaliação dos custos da qualidade na colheita florestal semimecanizada. Ciência Florestal, Santa Maria, v. 9, n. 1, p. 143-
160, 1999.

LEITE, H. G. et al. Determinação dos custos da qualidade em produção de mudas de eucalipto. Revista Árvore, Viçosa, v. 29, n. 6, p. 955-964, nov./dez. 2005.

MATOS, R. B.; MILAN, M. Aplicação sistêmica do modo de análise de falhas e efeitos (FMEA) para desenvolvimento de indicadores de desempenho de empresas de pequeno porte. Revista Árvore, Viçosa, v. 33, n. 5, p. 977-985, 2009.

MILAN, M; BARROS, J. W. D.; GAVA, J. L. Planning soil tillage using quality function deployment (QFD). Scientia Agricola, Piracicaba, v. 60, n. 2, p. 217-221, abr./jun. 2003.

MONTGOMERY, D. C. Introdução ao controle estatístico da qualidade. Rio de Janeiro: LTC, 2004. 513 p.

OAKLAND, J. S. Statistical Process Control. Oxford, England: Butterworth-Heinemann, 2003. $445 \mathrm{p}$.

PINTO, S. H. B.; CARVALHO, M. M. de; HO, L. L. Implementação de programas de qualidade: um survey em empresas de grande porte no Brasil. Gestão \& Produção, São Carlos, v. 13, n. 2, p. 191-203, maio/ago. 2006.

REZENDE, J. L. P. et al. Avaliação da qualidade na colheita florestal semimecanizada. Scientia Forestalis, Piracicaba, n. 57, p. 13 - 26, jun. 2000. STAMATIS, D. H. Failure mode and effect analysis: FMEA from theory to Execution. Wisconsin, USA: ASQ Quality Press, 1995. 495 p. TRINDADE, C. et al. Ferramentas da qualidade: aplicação na atividade florestal. Viçosa: UFV, 2000. 124 p. 